\title{
JORDAN AUTOMORPHISMS ON A SEMISIMPLE BANACH ALGEBRA
}

\author{
A. M. SINCLAIR
}

\begin{abstract}
In the group of (continuous) Jordan automorphisms, with the uniform topology, on a semisimple Banach algebra, we show that the connected component of the identity consists of automorphisms.
\end{abstract}

P. Civin and B. Yood have shown that a Jordan homomorphism (that is, a homomorphism that preserves the product $x \circ y=\frac{1}{2}(x y+y x)$ ) from a Banach algebra onto a semisimple Banach algebra is continuous provided the range algebra satisfies certain conditions $[1$, Theorem 4.7, p. 783]. These conditions may be reduced to the assumption that the range algebra is semisimple.

We sketch the proof and note its similarity to the proof of $[4$, Theorem 2]. Let $\theta$ be a Jordan homomorphism from a Banach algebra $A$ onto a semisimple Banach algebra $B$. Let $x_{n}$ tend to zero in $A$ and $\theta\left(x_{n}\right)$ tend to $y$ in $B$. Let $\pi$ be an algebraically irreducible representation of $B$ on a Banach space. Then $\pi \theta$ is a Jordan homomorphism from $A$ on to the primitive algebra $\pi(B)$. By a theorem of I. N. Herstein [3, Theorem H, p. 340] $\pi \theta$ is a homomorphism or an antihomomorphism. If $\pi \theta$ is an antihomomorphism, we consider $A$ with the reverse product. Therefore we may assume that $\pi \theta$ is a homomorphism of a Banach algebra on to the primitive algebra $\pi(B)$, and is thus continuous from $A$ into the Banach algebra of bounded linear operators on the representation space of $\pi$ by [4, Theorem 1]. This implies that $\pi(y)=0$. We now obtain the continuity of $\theta$ from the semisimplicity of $B$ and the closed graph theorem.

The Jordan automorphisms therefore form a topological group in the uniform topology as operators on the algebra with the composition of maps as multiplication. We are concerned with the connected component containing the identity in this topological group. We require the following lemma which is the analog for Jordan automorphisms and derivations of a theorem of G. Zeller-Meier for automorphisms and derivations [7, Théorème, p. 1131].

1. Lemma. Let $A$ be a Banach algebra and let $\alpha$ be a continuous Jordan automorphism on $A$. If the spectrum of $\alpha$ is contained in the open right

Received by the editors September 29, 1969.

AMS Subject Classifications. Primary 4650, 1740.

Key Words and Phrases. Semisimple Banach algebras, Jordan automorphisms, automorphisms, Jordan derivation, derivation, principal component of group. 
half plane $\{\lambda \in C: \operatorname{Re} \lambda>0\}$, then $\alpha$ is the exponential of a continuous Jordan derivation $\delta$ on $A$, and $\alpha$ lies in the uniformly continuous one parameter group $t \rightarrow \exp t \delta$ of Jordan automorphisms on $A$.

We shall not prove this result as the proof is the same as that of [7, Théorème, p. 1131], if we replace $G_{a}$ the left regular representation of $A$, by $J_{a}$ where $J_{a}(x)=a \circ x$. In the proof the associative formula $\delta(a b)-a \delta(b)=a^{\prime} b[7$, p. 1132] is replaced by $\delta(a \circ b)-a \circ \delta(b)$ $=a^{\prime} \circ b$, and there are similar changes in the other formulae involving products in the algebra. If $A$ has an identity 1 , in the proof we require $\theta(1)=1$ (if $A$ does not have an identity, we adjoin an identity and extend $\theta$ to the algebra with identity by defining $\theta(1)=1$ ). A Jordan automorphism on a semisimple Banach algebra preserves the commutativity of elements $[1$, p. 781], and hence is an automorphism of the centre of the algebra. This shows that $\theta(1)=1$.

2. TheOREM. Let A be a semisimple Banach algebra. Then the principal component in the group of Jordan automorphisms on A consists of automorphisms.

Proof. By Lemma 1 the Jordan automorphisms on $A$ of the form $\exp \delta$ where $\delta$ is a continuous Jordan derivation generate an open, and hence closed, connected subgroup $H$ of the group of Jordan automorphisms. Thus $H$ is the principal component of the group of Jordan automorphisms, and so to prove the theorem we have to show that $\exp \delta$ is an automorphism if $\delta$ is a continuous Jordan derivation on $A$. A continuous Jordan derivation on a semisimple Banach algebra is a derivation [6, Theorem 3.3], and the exponential of a derivation is an automorphism, so that the proof is complete.

3. Remark. We now outline an alternative proof of Theorem 2 when $A$ is a $C^{*}$-algebra. Let $\pi$ be an irreducible ${ }^{*}$-representation of $A$ on a Hilbert space $H$. Then $\pi(A)$ is a primitive algebra, and $\pi \theta$ is a Jordan homomorphism of $A$ onto $\pi(A)$. A theorem of $\mathrm{I}$. $\mathrm{N}$. Herstein [3, Theorem H, p. 340] implies that $\pi \theta$ is either a homomorphism or an antihomomorphism. If the dimension of $H$ is 1 , then $\pi \theta$ is a homomorphism. We now suppose that the dimension of $H$ is greater than 1 . Using a result of $\mathrm{R}$. V. Kadison [5, Theorem 1 , p. 274], [2, Lemma 2.8.2, p. 43] one can find a constant $k>0$ that is independent of $A, \pi$ and $H$ (of dimension $>1$ ) such that there are elements $x$ and $y$ of norm 1 in $A$ satisfying $\|\pi(x y-y x)\| \geqq k$. Let $\|1-\theta\|<k / 4$. We assume $\pi \theta$ is an antihomomorphism and suppose $x$ and $y$ of norm 1 have been chosen so that $\|\pi(x y-y x)\| \geqq k$. Then 


$$
\begin{aligned}
\|\pi(x y-y x)\| \leqq & \|\pi(x y)-\pi \theta(x y)\|+\|\pi \theta(y) \pi \theta(x)-\pi(y) \pi \theta(x)\| \\
& +\|\pi(y) \pi \theta(x)-\pi(y) \pi(x)\| \\
\leqq & \|1-\theta\|+\|1-\theta\| \cdot\|\theta\|<k
\end{aligned}
$$

so giving a contradiction. Thus every $\pi \theta$ is a homomorphism, and so $\theta$ is an automorphism.

\section{REFERENCES}

1. P. Civin and B. Yood, Lie and Jordan structures in Banach algebras, Pacific J. Math. 15 (1965), 775-797. MR 32 \#6245.

2. J. Dixmier, Les $C^{*}$-algèbres et leurs représentations, Cahiers Scientific, fasc. 29, Gauthier-Villars, Paris, 1964. MR 30 \#1404.

3. I. N. Herstein, Jordan homomorphisms, Trans. Amer. Math. Soc. 81 (1956), 331-341. MR 17, 938.

4. B. E. Johnson, The uniqueness of the (complete) norm topology, Bull. Amer. Math. Soc. 73 (1967), 537-539. MR 35 \#2142.

5. R. V. Kadison, Irreducible operator algebras, Proc. Nat. Acad. Sci. U.S.A. 43 (1957), 273-276. MR 19, 47.

6. A. M. Sinclair, Jordan homomorphisms and derivations on semisimple Banach algebras, Proc. Amer. Math. Soc. 24 (1970), 209-214.

7. G. Zeller-Meier, Sur les automorphismes des algèbres de Banach, C R. Acad. Sci. Paris Sér. A-B 264 (1967), A1131-A1132. MR 35 \#3443.

University of the Witwatersrand, Johannesburg, South Africa 